\title{
Optimizing Ages for Cervical Smear Examinations in Followed Healthy Individuals
}

\author{
Herbert P. Galliher, Ph.D.* \\ Department of Industrial and Operations Engineering, University of Michigan, \\ Ann Arbor, Michigan 48109
}

Presented at the Conference on Early Cervical Neoplasia, March 23-25, 1981

\begin{abstract}
A method is described and illustrated for calculating near optimal planned lifetime schedules of cervical smear examinations on an individual-by-individual basis. The method can be used to minimize the sum (discounted present value) of lifetime costs for prevention, management, treatment, and follow-up, plus the expected cost or loss if death occurs prematurely. A stochastic model of health, and of disease and death if they occur, is the basis of a computer program for finding the required examination ages. The program has built-in options of data and parameter values, which the user may override with preferred other values. The built-in data include estimates drawn from the British Columbia screening program, and, for loss due to death, from Social Security Administration data. Early cervical neoplasia is treated as being potentially nonprogressive to an extent that is unpredictable in the affected individual. Illustrations are presented of the comparative effects on optimal examination schedules, and on costs and return on preventive cytology regarded as an investment, of relatively low vs relatively high likelihood of disease onset occurring, of various levels of costs, including an effect of inflation, and of false-positive reports in cytology.
\end{abstract}

\section{INTRODUCTION}

Various investigators have made recommendations as to the age at which a female should have a first Pap-smear examination and how often she should have one thereafter. Gray has reviewed some of these recommendations [1] and we will not consider them here. Although the Pap test has been widely used in the belief that it is beneficial in the secondary prevention of cervical cancer, no significant trials have ever been mounted to evaluate it, and debate has continued as to its effectiveness. The probable benefit, and the frequencies for smear examinations that are needed to secure that benefit, have been estimated by taking what amount to hypothetical views about what happens when what the smear examination detects is, instead, undetected and untreated.

It would appear that the best frequency plan might well differ, even significantly, from individual to individual. Considering the etiological evidence, individuals could be expected to differ in their percêptions of their own risk levels for cervical cancer. The fact that knowledge about cervical neoplasia is still incomplete, that trends may be occurring in the likelihood of developing the

* Current address. 
disease, and that new methods and criteria of diagnosis and of management are continuing to develop, can be expected to have different significance for different individuals. These, again, cannot be expected to have the same objectives, nor the same resources, for this preventive aspect of personal health, nor to have the same perception of the threat which cervical cancer poses to their bodies and lives, nor are they likely to be expecting to suffer the same loss if the disease does develop, nor to expect their survivors to suffer the same losses if they die from it prematurely. Yet these considerations all go into a rational individual decision as to how intense prevention to undertake.

This report summarizes and illustrates briefly a computerized approach that has been developed with the aim of permitting factors and considerations of the above sort to be taken into account on an individualized basis and to calculate, for any healthy such individual (or category of such individuals) a planned schedule of ages for her successive smear examinations. The computer program has built-in default options for the input factors, but allows the user to override these with preferred other values. It produces approximately optimal schedules readily, so that the user can deliberately vary the inputs in multiple computer runs in order to observe how sensitive the resulting schedules are to possible unreliability of the input assumptions. The general approach is based upon a stochastic representation of health, cervical neoplasia and cancer, and death from other causes. It attempts to utilize views concerning the disease that are acceptable in gynecologic oncology. One optimal mode is straightforwardly actuarial, and might be developed into a basis for insurance against death from cervical cancer. The program produces data of potential epidemiological interest.

\section{PARAMETERS, DATA, AND METHODS}

The computer program offers as options certain baseline values for parameters and data sets, but the user can employ other values of his choosing.

\section{a. Disease, Detection and Behavior}

(1) Stochasticity. The program steps an individual through life in a stochastic fashion in 3-month time steps, starting with the individual at age 20 and healthy, and ceasing to follow healthy individuals at age 80. At each such step an outcome is assigned, e.g., for a healthy individual the outcome will be either to remain healthy, or to die of causes that have nothing to do with cervical cancer, or to develop an early phase of neoplastic disease. Outcomes are assigned randomly, in accordance with probabilities that can depend upon the age and state of the individual. Equivalently, the program may be thought of as stepping a large number of individuals who are identical as to age and state, with the number that are assigned to each outcome being that which is on the average expected in accordance with the associated probabilities.

(2) Sirains. For the illustrations presented below it is assumed that there are two significant kinds, or strains, of early neoplasia, of which any given individual can develop at most one. One strain, termed "progressive," will sooner or later become invasive cancer in the affected individual if it is not treated. The other, termed "nonprogressive," will never so progress. The program can be readily modified to provide for any number of strains each of which has an early phase 
and an invasive phase, or either alone, provided that strains are so defined that only a negligible number of individuals would develop more than one strain during life; computing time is proportional to the number of strains.

(3) Biological onset. For each strain the user must select a rate of biological onset (probability of onset at each time step) of the early phase, which may be age specific. A built-in option follows the age distribution of the incidence rates of carcinoma in situ in followed individuals reported from the British Columbia program in 1968 [3]. Those rates are highest during the period from Ages 20 to 35 , declining progressively thereafter at a decreasing rate. In the illustrations below this same distribution has been used for both the progressive and the nonprogressive strain, but with different overall rates. In compiling Table 2 we observed that the total incidence rate of diagnoses of dysplasia was approximately $54 \%$ of that for carcinoma in situ. In the illustrations the biological onset rate for the nonprogressive strain has been set at $54 \%$ of that for the progressive strain, at each age step. Onset rates can be entered by multiplying their age distributions by desired constants. In this way various levels of risk for the disease, or epidemiologically projected population rates, can be represented.

Onset of an early phase is treated as being initially occult in the affected individual, with detection and diagnosis occurring, perhaps imperfectly, as described below.

(4) False-positive results of examinations. When the effectiveness of scheduled cytology in preventing advanced cancer is being evaluated, any smear that is reported as abnormal and possibly signifying early neoplasia, but which in fact does not, functions as a "false-positive." Varying degrees of follow-up are then required in order to rule disease out. In the computer program such developments do not become part of the history of an individual. The total average incidence rate in Table 1 is approximately 16.4 times that in Table 2 . In the illustrations this ratio has been used, except as noted, at each age step at which preventive cytology is scheduled, to generate an expected complement of false-positive load for cytology. This load thus constitutes an involuntary additional investment in cytology.

(5) Age-specific death rates from other causes. The program contains a builtin age-specific table of death rates from causes other than cervical cancer, which was developed from 1965 United States mortality data. An option allows the user to assign a multiplicative factor, e.g., risk level, for such death.

(6) Probability distribution of the natural duration of early disease. The natural duration of early disease, i.e., its duration in the absence of detection or intervention, is assumed to be random in any affected individual. For each strain the program allows the user to specify a particular value for the average length of this duration. However, the form of the probability distribution of this duration is assigned to be the same for every strain, namely, that of the (negative) exponential probability distribution. Thus the distribution is in each case completely determined simply by specifying the mean (average) duration. For example, suppose that the early phase of one strain is being used to represent carcinoma in situ. Then given that a represented individual has unsuspected carcinoma in situ at some point in time during her life, the probability, after a further duration 
of time of length $t$, that this condition is still present and has not progressed to invasive disease in the interim, assuming that it has not been detected or treated in that interim, is equal to $e^{-v r}$. Here the mean duration is equal to $1 / v$.

For reference, the typical numerical implications of this particular distribution with a mean of 10 years are suggested by the following set of sample values:

$\begin{array}{lrrrrrrr}t \text { (years) } & 0.5 & 1.0 & 2.0 & 5.0 & 10 . & 20 . & 30 . \\ e^{-v t}(\%) & 95 & 90 & 82 & 61 & 37 & 14 & 5\end{array}$

Thus $5 \%$ of all cases would be postulated to progress within 6 months or less after onset, or after any stipulated point in time (and about $1 \%$ in a little over a month) while at the other extreme $5 \%$ would be capable of going for 30 years or more without progressing. The illustrations below all assume a 10-year mean duration for the progressive strain.

In the program as it is presently constructed, it is assumed that the value of $1 / v$ is not affected by the age of the diseased individual. A strain which is such that early disease is never of the progressive sort is readily represented by setting the value of $v$ equal to 0 . In order to represent a strain which never presents itself (pathologically) as early disease, one simply sets the value of ' $v$ equal to a large number, so that, in effect, the mean duration of early disease is equal to 0 .

(7) Noncytological detection of early disease. Since the intent of the program when used in certain modes is to optimize cytology which has been previously scheduled, it is important to account for cases which are detected at other times, and such cases are referred to as having been noncytologically detected. For example, some complaint may have caused the individual to seek medical examination at other than the time for a regularly scheduled cervical smear, as a result of which-albeit with the assistance of cytology-the disease was detected. The reason may be of any sort. The program assumes that such detection could only occur randomly, and independently of any specific influences. For a case which is in the early phase, and which is eligible for such detection for a time period of length $t$, it is thus assumed that the probability that such detection does not occur is equal to $e^{-b t}$. Here it is natural to consider the quantity $b$ as being the "rate" at which such detection occurs. In the absence of all preventive cytology, the fraction of cases which would be detected noncytologically, if the numerical effects of the occurrence of death from other causes is ignored, is equal to $b /(b+v)$. (We omit the mathematical argument, which is well known.) This may be used to set the numerical value of $b$ in relation to the value being used for $v$. Note that the value of $b$ is not affected by the frequency of scheduled smear examinations. Except as noted, in the illustrations below $b$ has been taken to be equal to 0 .

(8) Invasive disease and its noncytological detection. For any strain that has an invasive phase, the program assumes that time is of the essence in detecting invasion if death is to be avoided. Thus invasive disease is assumed to be curable at its onset, but ultimately to convert to disease which at detection will (retrospectively) be found to be incurable. Once onset occurs, an ongoing competition is thus assumed to be initiated between detection and such conversion. 
Noncytological detection can now include classical "clinical" detection, viz., from signs and symptoms. Both noncytological detection and (virtual) conversion to incurable (fatal) disease are assumed to be events which occur randomly during time (if at all). The time rates at which these events tend to occur are taken to be constant and independent of the age and the history of the affected individual, but can differ from strain to strain. Thus, given that an individual unsuspectedly has the invasive phase of some strain at some point in time during her life, the probability, after a succeeding duration of time of length $t$ during which cytological detection does not occur, that the condition has neither become incurable nor been noncytologically detected, is assumed to be of the exponential form $e^{-m t} e^{-g^{t}}$, where $m$ is the time rate for conversion to fatal disease and $g$ is the time rate for noncytological detection. Equivalently, for disease that is not subject to cytological detection, the quantity $1 / g$ is the average age of invasion (since onset) at noncytological detection, while $1 / \mathrm{m}$ is its average age in undetected cases at the time that conversion to fatal disease occurs.

The user may assign values to $g$ and $m$. In the illustrations below we have used $1 / g=3 \frac{1}{3}$ years. If cytological detection were not available-as was the case historically before the Papanicoulaou smear-then of detected cases the fraction that would be found to be curable would be equal to $g /(g+m)$ if the numerical effects of intervening death from other causes is ignored (again we omit the mathematical argument). In the illustrations we have taken this to be equal to $54 \%$, so that $1 / m$ becomes, roughly, 4 years. It must be kept in mind that these parameter values represent averages. For reference, the following table of sample values profiles the numerical implications of these particular assumptions (again ignoring the effect of death from other causes):

\begin{tabular}{|c|c|c|c|c|c|c|c|c|c|}
\hline $\begin{array}{l}\text { Age of invasion since } \\
\text { onset (years) }\end{array}$ & $1 / 6$ & 0.5 & 1.0 & 1.5 & 2. & 3. & 4. & 5. & 7. \\
\hline $\begin{array}{l}\text { Percentage not yet } \\
\text { detected if no } \\
\text { cytology }\left(100 e^{-g t}\right)\end{array}$ & 95 & 85 & 72 & 61 & 51 & 37 & 26 & 19 & 10 \\
\hline $\begin{array}{l}\text { Percentage curable if } \\
\text { detected at this } \\
\text { age }\left(100 e^{-m t}\right)\end{array}$ & 96 & 88 & 78 & 69 & 61 & 47 & 37 & 29 & 17 \\
\hline
\end{tabular}

(9) Compliance, false-negative rate. An individual may fail to show up for a scheduled cervical smear examination, or at such an examination disease which is present may fail to be detected. In either case disease can be missed. The program requires that a probability, the miss probability, be assigned a numerical value. This value is applied to each scheduled examination, regardless of the age or history of the individual examined. In the illustrations below we have assumed that the no-show probability is $20 \%$ and the false-negative rate is $15 \%$, resulting in a miss probability of $1-(0.8)(0.85)=32 \%$.

\section{b. Costs}

(1) Inflation (deflation); discounting. While the program contains built-in estimated cost values for use as "default" options, the user can prefer other values, 
e.g., in higher-cost applications or when the cost of premature death would be much higher (or lower) than the approximate average for the United States. The present program assumes that all unit-cost values will vary historically, during the planning future, only in accordance with inflation (or deflation) at a constant time rate. Accordingly, input unit-cost values should be stated in current dollars, and in the illustrations below we have used estimated 1981 values.

For cost optimizations the user must specify a postinflation discount factor, the value of which might vary considerably depending upon the application since its function is to reflect the available postinflation return on investment from alternative opportunities (personal, medical, economic). Note that the agewise increase in the rate of death from other causes provides an inherent biological discounting effect, at an increasing rate. Although the effect of discounting is to make the resulting calculations of optimal strategies be less sensitive to errors in input values and to invalidity of assumptions, this effect should be reduced by inflation and augmented by deflation. In Tables 3-6 we have used a postinflation discount rate of $4 \%$, primarily because the estimated U.S. values for cost of premature death were not available at a lower rate.

(2) Cost of a cervical smear. This may vary widcly depending upon the application. An excessive cost can make it cost optimal to forego preventive cytology entirely. For a smear obtained during a multiphasic physical examination this cost should include the proportionate share not only of the time for that part of the examination but for the patient's indirect cost as well. In the illustrations presented below we have used $\$ 9$.

(3) Costs of management, treatment, and follow-up. These should include indirect costs. For each phase of each strain the program requires that a cost value be specified which equals the discounted present value, at the time detection occurs, not only of treatment but for all follow-up of the individual from then on. The latter is because the computer model provides no further stochastic tracking of a detected individual, and because it is assumed that strains have been defined in such a way that no individual can develop more than one strain. Since the effect of postinflation discounting is to dampen the contribution from such later follow-up expenses, nominal values or rough estimates of these later costs may suffice, although the extent to which resulting errors may affect optimal strategies may be best assessed experimentally.

In the illustrations below we have employed the following values in terms of 1981 dollars, and these are built-in program options:

Average cost of management, treatment, and

Condition follow-up (discounted)

Insignificant + smear

Nonprogressive early disease

Progressive early disease

Curable invasive disease

Incurable invasive disease
$\$ 100$

2,000

3,000

5,500

17,000 
Since the modalities with which these conditions are treated varies considerably, obviously a single figure for cost has to be an average.

(4) Costs due to premature death. Premature death is no doubt the outstanding threat posed by cervical cancer. No qualitative index of prematurity appears to have been established. But estimates of the cost, in terms of lost expected subsequent earnings, have been published by Cooper and Rice at the Social Security Administration, as of 1972, averaged for U.S. females [4]. Their estimates include the marketplace equivalent of housewifely duties, and are discounted present values assuming no inflation after death. Applying the $97 \%$ increase in the consumer price index that has occurred from 1972 through 1980 , but without adjusting for any interim change that may have occurred in relative participation in the workforce by females, their estimates in the case of $4 \%$ discounting become:

\begin{tabular}{lc} 
Age & $\begin{array}{c}\text { Present value of subsequent ex- } \\
\text { pected earnings }\end{array}$ \\
\hline $20-24$ & $\$ 235,843$ \\
$25-29$ & 227,787 \\
$30-34$ & 208,744 \\
$35-39$ & 187,412 \\
$40-44$ & 163,499 \\
$45-49$ & 136,528 \\
$50-54$ & 106,222 \\
$55-59$ & 74,828 \\
$60-64$ & 46,630 \\
$65-69$ & 25,712 \\
$70-74$ & 14,075 \\
$75-79$ & 7,318
\end{tabular}

A smoothed version of this table is available in the computer program, together with the option to multiply all of the above values by a desired constant, e.g., to adjust for individuals whose projected incomes are expected to be significantly different from the U.S. average. The discount and inflation factors described earlier are applied to the above table values, but not to the earnings rates upon which the Cooper and Rice tables are based; for them, the $4 \%$ after-death discounting without inflation remains unadapted in our program.

\section{c. Optimization Procedure}

For a 20-year-old individual, a cost-optimal schedule of planned examination ages is one that minimizes the total discounted present value, at age 20 , of all cxpcctcd futurc costs. This total is the equivalent required reserve to be invested at age 20 at an interest rate equal to the discount rate, to meet such costs, a standard concept in financial maintenance planning. To find the exactly optimal ages is relatively easy, with a computer, using a mathematical technique known as dynamic programming, provided that the miss probability is zero. If the miss 
probability is greater than zero, the task can become relatively expensive of computer time, since no simple mathematical method short of exhaustive search is known in that case. A limited such search routine is available within the computer program. It has not been used in the illustrations presented below, and the "required ages" given there assume that the miss probability is zero. However, for those schedules, the "total costs" in the tables are the correct ones when the assumed miss probability (e.g., 32\%) is used in the calculations.

The optimization can be started at any age with the individual known to be free of disease at that age. For all the illustrations below, this age has been taken to be 20 years.

\section{ILLUSTRATIONS AND DISCUSSION}

The rates in Tables 1 and 2 were compiled from histories of each of the individuals in the two birth cohorts who were seen in the British Columbia program during the years 1950-1969, being some 120,000 persons in all. The histories were made available to us on tape by the program's investigators. In addition to containing the year and month of birth of the individual, each entry in her history was dated as to year and month and was either a report of a smcar examination, a report of a pathological investigation, a report of treatment (by type), a report of death with cause as cervical cancer where known, or some

TABLE 1

Incidence of First Abnormal Smear in Followed Individuals ${ }^{a}$

\begin{tabular}{|c|c|c|c|c|c|c|}
\hline \multirow[b]{2}{*}{ Year } & \multicolumn{3}{|c|}{ Born 1914-1918 } & \multicolumn{3}{|c|}{ Born 1929-1933 } \\
\hline & $\begin{array}{l}\text { Average } \\
\text { age }\end{array}$ & $\begin{array}{l}\text { Person- } \\
\text { years }\end{array}$ & $\begin{array}{c}\text { Rate per } \\
1000\end{array}$ & $\begin{array}{l}\text { Average } \\
\text { age }\end{array}$ & $\begin{array}{l}\text { Person- } \\
\text { years }\end{array}$ & $\begin{array}{c}\text { Rate per } \\
1000\end{array}$ \\
\hline 50 & $34)$ & & & $19)$ & & \\
\hline 51 & 35 & & & 20 & & \\
\hline 52 & 36 & 994 & 25.2 & 21 & 298 & 16.8 \\
\hline 53 & 37 & & & 22 & & \\
\hline 54 & 38 & & & 23 & & \\
\hline 55 & 39 & 1,147 & 15.7 & 24 & 498 & 10.0 \\
\hline 56 & 40 & 1,984 & 13.1 & 25 & 930 & 9.7 \\
\hline 57 & 41 & 3,044 & 8.5 & 26 & 1,536 & 11.1 \\
\hline 58 & 42 & 4,492 & 9.6 & 27 & 2,476 & 7.3 \\
\hline 59 & 43 & 6,758 & 8.4 & 28 & 4,252 & 5.4 \\
\hline 60 & 44 & 9,594 & 9.7 & 29 & 6,859 & 8.2 \\
\hline 61 & 45 & 13,388 & 9.3 & 30 & 10,638 & 7.3 \\
\hline 62 & 46 & 17,525 & 7.9 & 31 & 16,072 & 8.0 \\
\hline 63 & 47 & 21,294 & 9.1 & 32 & 21,716 & 8.7 \\
\hline 64 & 48 & 23,994 & 13.3 & 33 & 26,566 & 12.9 \\
\hline 65 & 49 & 26,016 & 9.9 & 34 & 30,606 & 11.9 \\
\hline 66 & 50 & 27,586 & 11.2 & 35 & 33,939 & 13.3 \\
\hline 67 & 51 & 28,534 & 15.8 & 36 & 36,616 & 19.2 \\
\hline 68 & 52 & 28,854 & 15.7 & 37 & 38,158 & 21.0 \\
\hline 69 & 53 & 28,584 & 19.4 & 38 & 38,382 & 29.3 \\
\hline
\end{tabular}

${ }^{a}$ In the British Columbia screening program. 
TABLE 2

Incidence of Dysplasia or Stage 0 in Followed Individuals ${ }^{u}$

\begin{tabular}{|c|c|c|c|c|c|c|}
\hline \multirow[b]{2}{*}{ Year } & \multicolumn{3}{|c|}{ Born 1914-1918 } & \multicolumn{3}{|c|}{ Born 1929-1933 } \\
\hline & $\begin{array}{l}\text { Average } \\
\text { age }\end{array}$ & $\begin{array}{l}\text { Person- } \\
\text { years }\end{array}$ & $\begin{array}{c}\text { Rate per } \\
10,000\end{array}$ & $\begin{array}{l}\text { Average } \\
\text { age }\end{array}$ & $\begin{array}{l}\text { Person- } \\
\text { years }\end{array}$ & $\begin{array}{c}\text { Rate per } \\
10,000\end{array}$ \\
\hline 50 & $34)$ & & & 19 & & \\
\hline 51 & 35 & & & 20 & & \\
\hline 52 & 36 & & & 21 & & \\
\hline 53 & 37 & & & 22 & & \\
\hline 54 & 38 & 18,910 & 5.8 & 23 & 10,130 & 3.0 \\
\hline 55 & 39 & & & 24 & & \\
\hline 56 & 40 & & & 25 & & \\
\hline 57 & 41 & & & 26 & & \\
\hline 58 & 42 & & & 27 & & \\
\hline 59 & 43 & & & 28 & & \\
\hline 60 & 44 & 9,844 & 3.0 & 29 & 6,945 & 4.3 \\
\hline 61 & 45 & 13,772 & 5.1 & 30 & 10,290 & 3.9 \\
\hline 62 & 46 & 18,081 & 5.0 & 31 & 16,235 & 8.0 \\
\hline 63 & 47 & 22,044 & 6.4 & 32 & 22,196 & 6.8 \\
\hline 64 & 48 & 25,061 & 6.4 & 33 & 27,178 & 18.0 \\
\hline 65 & 49 & 27,492 & 7.6 & 34 & 31,554 & 15.8 \\
\hline 66 & 50 & 29,461 & 10.2 & 35 & 35,240 & 15.3 \\
\hline 67 & 51 & 30,940 & 9.4 & 36 & 38,494 & 12.7 \\
\hline 68 & 52 & 31,892 & 7.5 & 37 & 40,836 & 11.5 \\
\hline 69 & 53 & 32,328 & 6.8 & 38 & 42,022 & 17.6 \\
\hline
\end{tabular}

${ }^{a}$ In the British Columbia screening program.

combination of these. We wrote a computer program to process, from these histories, various "first-event" rates, by following individuals who were at risk for the event. The rates in Tables 1 and 2 were calculated assuming that the individuals at risk are still being followed after the last entry in their histories.

During the 1950s the risk pool was too small to provide significant yearly rates. During the 1960s a marked increase is evident in rate with age and year of observation, for each birth cohort, as is also a difference between the two cohorts. By comparison, the age-specific incidence rates (per 10,000 person-years) for carcinoma in situ reported by Fidler et al. ([3], Table XI), when all women screened in the program in the years 1960-1966 are pooled, average between 8.1 and 11.9 from ages 20 to 35 , then decline progressively to about 2 by age 75 .

It is not our task here to interpret the apparent trends in Tables 1 and 2, nor to extrapolate an estimated update of the rates to 1981 and beyond. What these data suggest is that it would appear to be of interest to include, in our illustrations, a range in the level of values of onset rate (and these can of course be expected to exceed observed incidence rates) that would be considered as representing an average level of risk for cervical cancer. We have selected three levels, corresponding to age-adjusted rates of onset (onsets per 10,000 person-years) of the progressive strain of 4.0 (Table 4A), 11.4 (Table 3), and 29.0 (Table 4B). In 


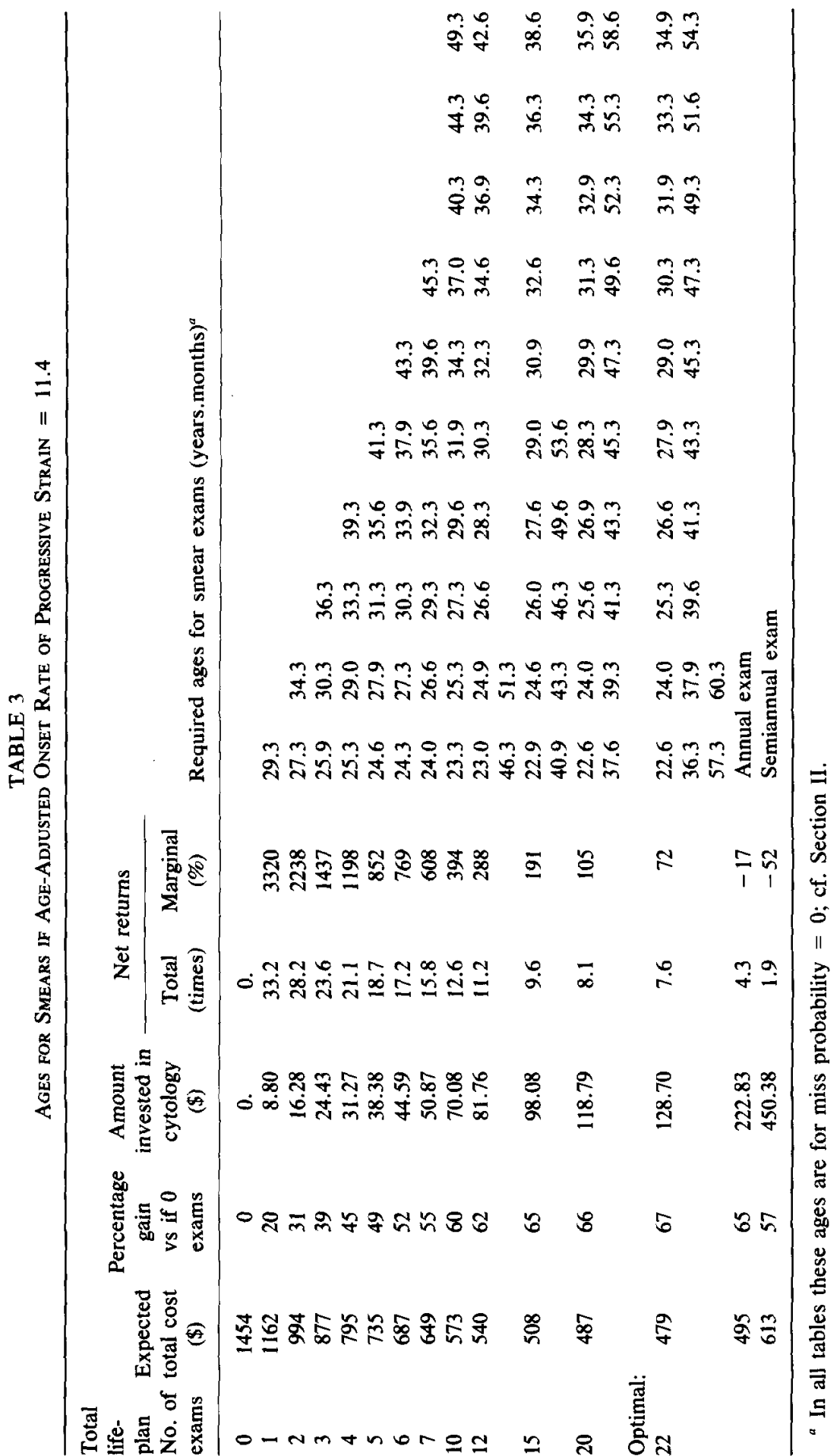




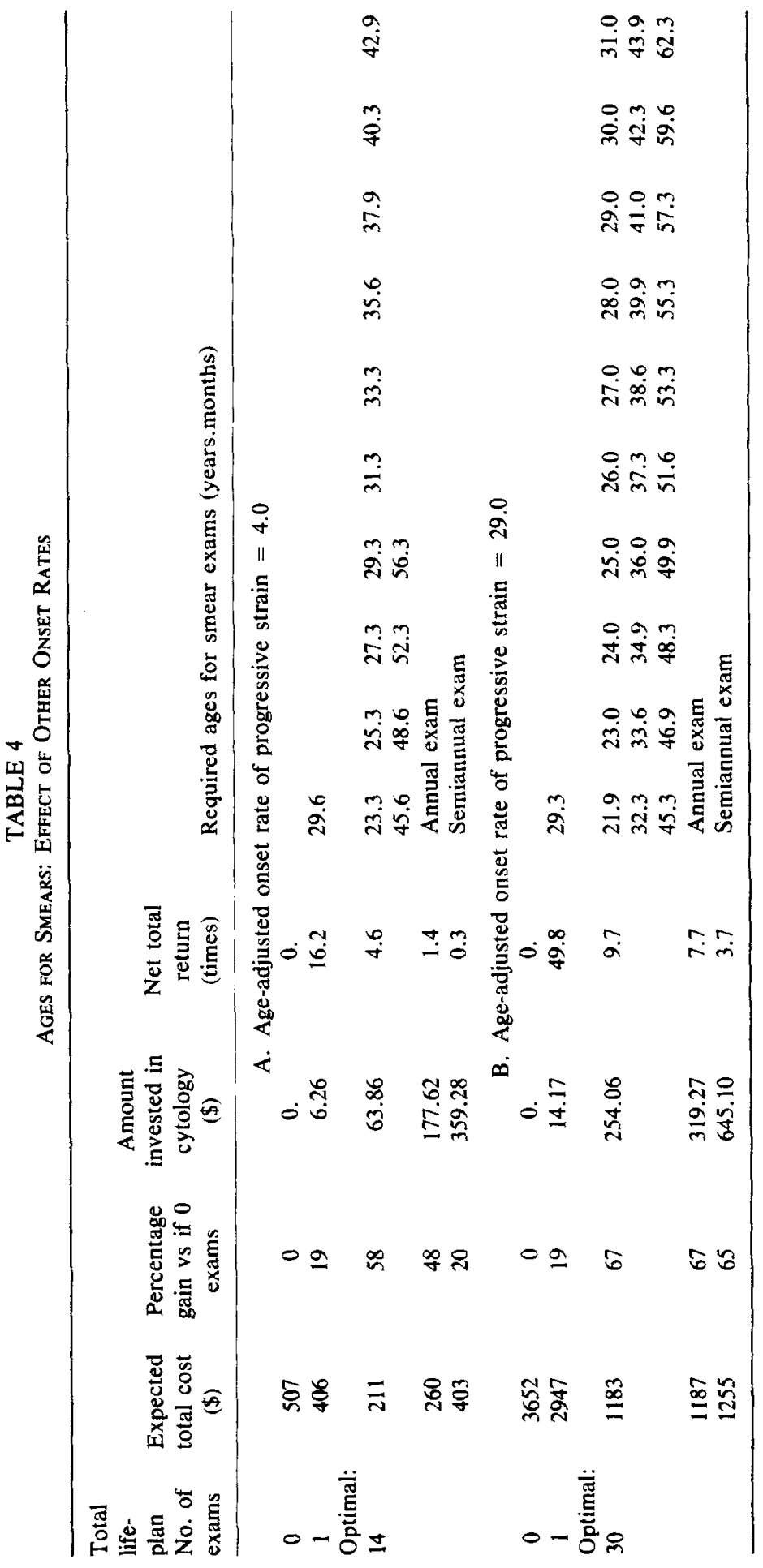


any given application, of course, the appropriate rate might or might not fall within this range.

Table 3 is an extract of the information that is routinely produced by a computer run. The best "life-plan number of exams" is found (by the program) by examining each possible number, from 0 , which represents no use of cytology at all, up to a number which is sufficiently large that the optimum number has been found. For brevity's sake only selected values of the possible number of examinations are included in the table. The "expected total cost" includes the amount invested in cytology, which is shown separately. The optimum plan is one that minimizes this total, which in this case is 22 examinations. For each planned number of examinations the reduction in the baseline total cost, namely that for no planned cytology, is shown, and in this case is $67 \%$ at the optimum. Cytology can be regarded as an investment, and the total return on it at the optimum is $760 \%$. Although the "marginal" return on investment would normally be calculated by comparing, for example, 20 examinations with 19 , we have calculated it from one row in the table to the next lower row; thus when the number of planned examinations is increased from 15 to 20 , there is a $105 \%$ return on the additional investment in cytology.

It can be seen that to achieve the optimum cost the 22 examinations should be done at about every 15 months to age 35 , the interval lengthening gradually thereafter. By comparison, an annual examination is nearly as efficient, although the cytological workload is nearly double, at a not quite offsetting reduction in other costs, viz., a $17 \%$ marginal loss from the optimum, which is comparatively small.

To save space only the principal features and results for the other two values of onset rate for the progressive strain are shown, in Table 4. For the lower rate, the maximal attainable return on cytology, as a percentage of the baseline, is now lower. The annual smear does not compare as favorably. If only one examination were to be done, it should be done at about the same age as in Table 3. For the higher onset rate, the optimal examination frequency is virtually an annual one. Discounting dampens the contribution of events of later years to present cost, so that it is not the examinations past the age of 70 , say, that make the difference between the annual and the optimal plans in this case, but, rather, the other differences in their schedules.

These tables illustrate that even if only one examination is done in a lifetime, the baseline burden will be reduced markedly if that examination is done at the right age. Similarly, a few well-timed examinations are very effective, and have large marginal incentives. The program permits one to schedule any given number of examinations so as to minimize the probability that invasive disease ever develops in the person at risk (whether it is ever detected or not). Table 5 shows plans for doing this. There can now be no optimum, since the more examinations the lower the probability (thus this type of information, while perhaps reassuring, is not sufficient for resolving competing medical priorities). From this table we see that so-called "unscreened" individuals, unless they have never had so much as one Pap smear done, may well show an incidence rate of invasive disease that is lower than might be presumed. Note that in this table we are dealing 


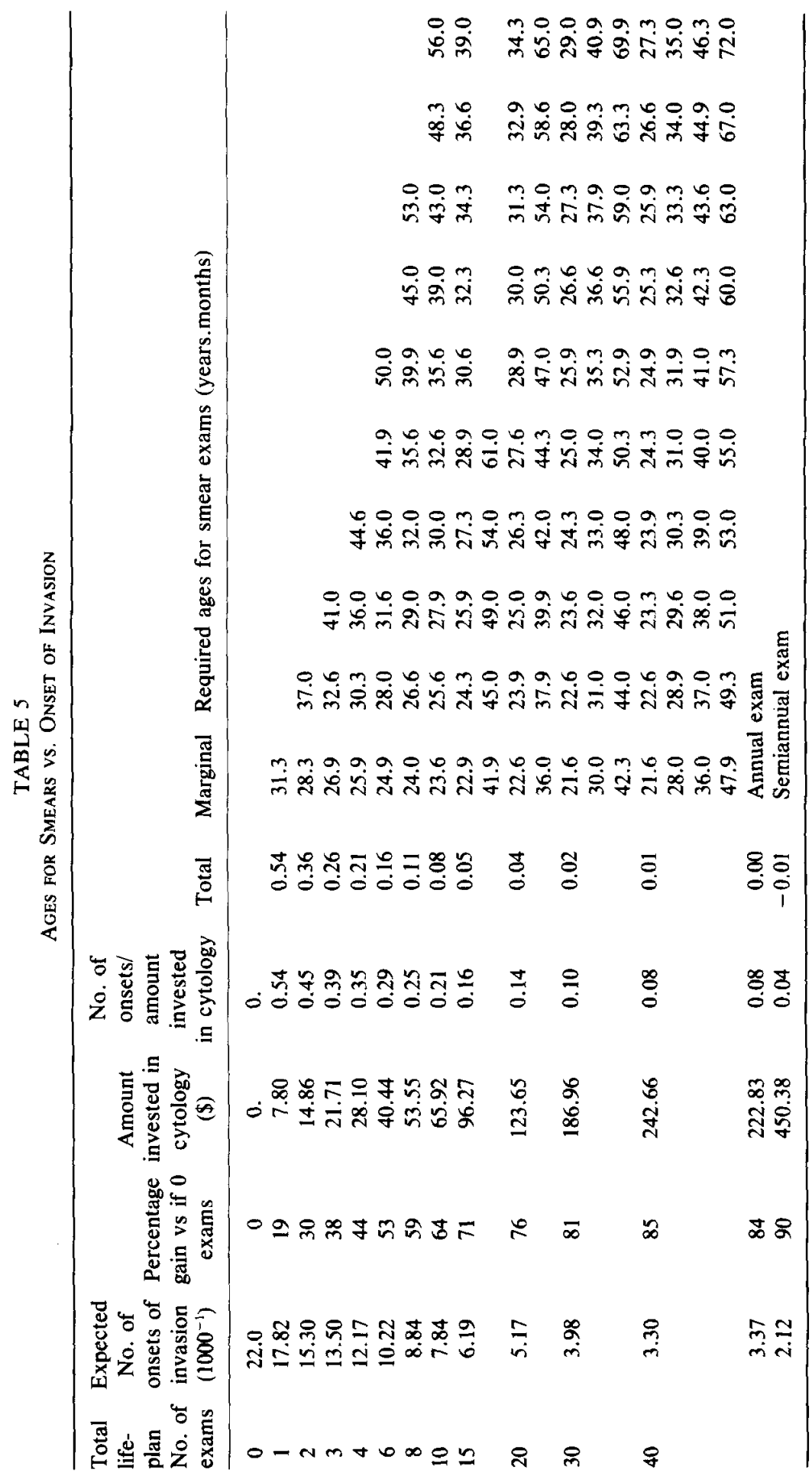


with number of biological onsets, not (clinical) incidence, of invasion. Number of onsets has not been discounted, but cytological costs have.

In the case of individuals whose income level is higher than the U.S. average, the cost of premature death could be higher than that used for Table 3. In Table 6 are the results when this cost is assumed to be five times the national average, under the assumption that all other costs remain the same. The potential for cytology is now much greater. Optimal smear frequency, viz., every 9 months to age 35 , is not proportionately greater.

During the years that we have studied the subject of this paper, progressive inflation has repeatedly made it necessary to revise cost data tables upward. Severe inflation tends to create a current shortage of capital and to reduce the competitiveness of the return on other than the most critical or favorable investments. If inflation is severe enough, the effective postinflation discount rate may even become negative. For Table 7 it has been assumed that this rate has been reduced to zero. Examination ages now tend to be later than those in Table 3. The investment return from preventive cytology appears as having increased, as has the optimal cytological workload, although the attainable efficiency has dropped. To have inflation make profitable investments appear to be even more so can be an undesirable unbalancing effect.

The various tables suggest how the conclusions from computer runs are sensitive to the values of certain of the input factors. The following additional observations are based upon other computer runs whose results are not presented in detail here.

(1) If the cost of the smear examination were $\$ 15$, in Table 3 the optimal early interval between scheduled examinations would increase to 18 months, with the optimal total number of examinations dropping to 18 and the optimal percentage gain dropping to $62 \%$. Thus, there is a significant incentive for having the cost of the smear examination (the total cost) be as low as possible, including the fact that more disease will be prevented when cytology is then optimally utilized. In Table 6 , had the examination cost been $\$ 30$, with a comparable increase in false-positive costs, the optimal early interval would have increased to 15 months, with the total planned number of examinations decreasing to 26 and the optimal percentage gain decreasing to $78 \%$. As is suggested by Table 3 , the cost of the smear examination would have to approach toward $\$ 300$ before the result would be that scheduled cytology would become unprofitable, yet presumably its attractiveness would have been lost long before that cost level had been reached.

(2) The effect of the cost of resolving false positives is, it would seem, to increase the virtual cost of the smear examination itself. Doubling the falsepositive rate, or its cost, increases the optimal early interexamination interval from 15 to 18 months, although still with 21 examinations in all being optimal, while the cytology cost is increased by about $33 \%$. Had it been assumed, for Table 3, that the false-positive rate was zero, the age schedules would not have changed significantly, although the optimal total planned number of examinations would have decreased to 19 while the optimal percentage gain would increase to $72 \%$.

(3) When only a few examinations are planned per lifetime, achieving a $0 \%$ 
m.

mommam ammm

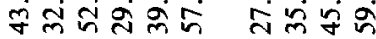

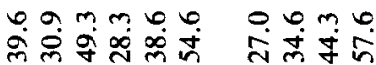
moำm mama

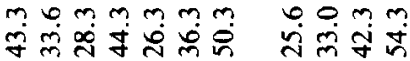

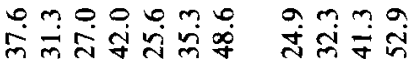

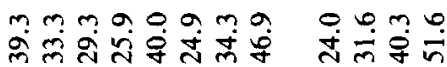

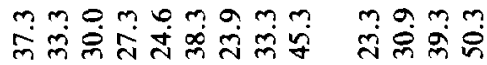

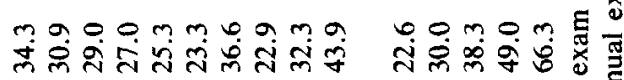

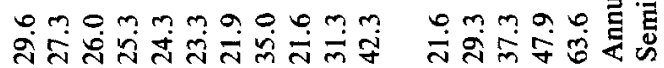

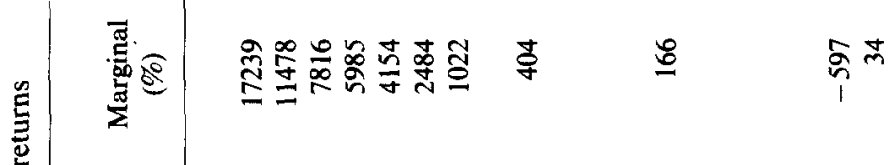

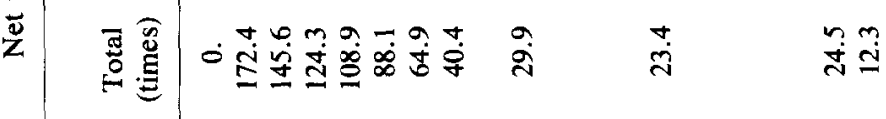

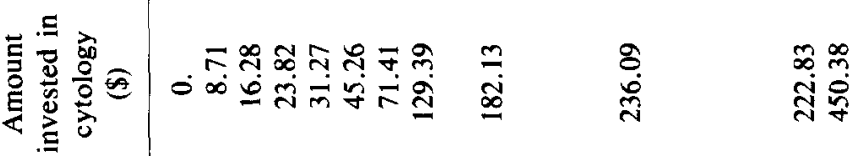

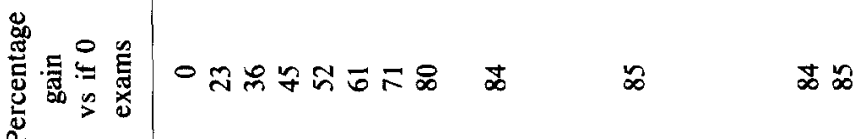

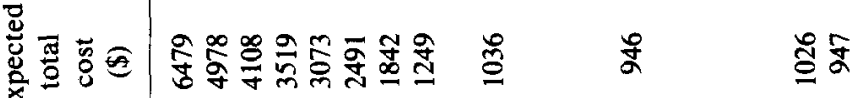
舀

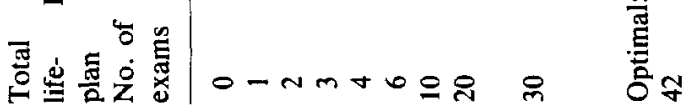




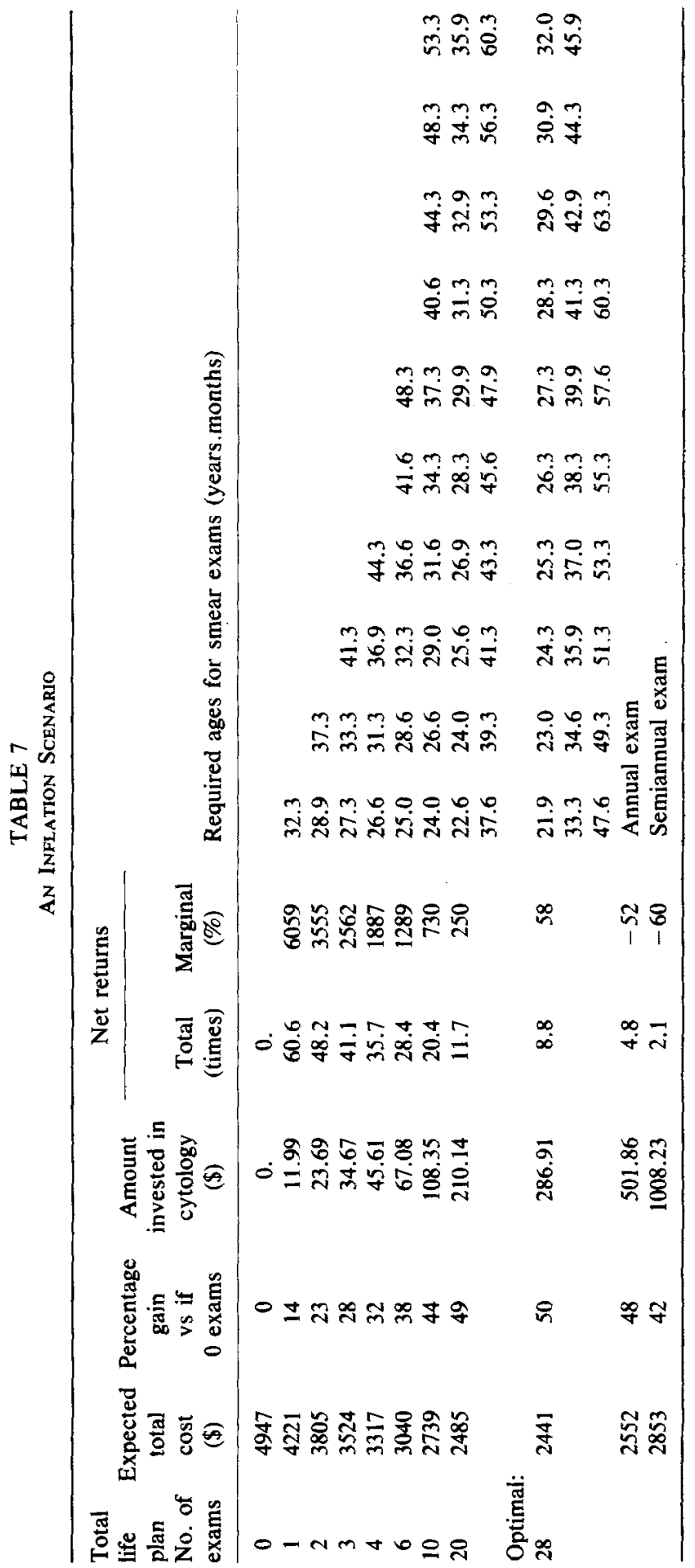


false-negative rate can have a significant effect on percentage gains and returns, but a lesser effect on schedules, to judge from a fcw exploratory exhaustive searches that we have made. But when examinations are done fairly frequently, as is typically the case for the "optimal" plans in the tables, the effect can be expected to be relatively smaller, at least if, as is assumed, disease that is present is not more likely to be missed at subsequent examinations if it was missed on any one occasion. This is because, even with a miss probability of $32 \%$ per occasion, only $10 \%$ will have been missed after two scheduled examination times have passed, only 3.3\% after three, and so on. The implications when such interexamination "independence" cannot be assumed have not been studied. Otherwise, when preventive cytology is optimally used, there would not appear to exist a marked incentive for having an examination technique that yielded a lower false-negative rate, nor for having a sharply greater show probability, than has been assumed.

(4) In the case of Table 3, if it were assumed that $67 \%$ of early-phase disease is self-detectable (as defined above), the optimal total planned number of examinations would not change significantly, nor the schedule for them, but the percentage gain at the optimum would drop to $60 \%$. But were the self-detectable percentage to be as large as $85 \%$, the optimal total planned number of examinations would decrease to 17 , the early interval now being 18 months, with the baseline burden dropping to $\$ 844$. More interesting in that case is the fact that total costs, at the optimum, would be $\$ 427$ vs $\$ 479$, viz., still roughly comparable.

In a similar way the computer program can be used to develop answers to other questions, such as the effect on conclusions were the age distribution of onset rates different from what has been assumed in the above illustrations; or were the durations of early disease, or of curable invasion, or of undetected invasion, assumed to be quite different than has been assumed here; or, were alternative detection techniques to be posed for comparative evaluation; or were management costs to be significantly different from the values that have been used in the illustrations here.

The general approach lends itself to studying alternative hypotheses, from those underlying the present program, as regards the nature of risk, the variety of disease, potential time shifts in onset rates, age specificity of the values of disease parameters, the degree of multidimensionality of onsets and of terminations of disease phases, the behavior of individuals at risk, and other aspects.

\section{SUMMARY}

It has not been the objective of this paper to make recommendations as to when females should have Pap smear examinations. We have described a method that would appear to facilitate making this decision readily on an individualized basis while yet taking advantage of epidemiological information. The method has not been tested in such use. We have illustrated the results in the case of a few selected values of the required input information that should be of interest, including illustrating degrees to which the decision would be sensitive to the choice of the values assigned to various of the input factors.

It seems to us that the promise of being able to make the decision on an 
individual basis but in a formal manner that explicitly synthesizes the relevant factors and considerations, will increase the demand for the kind of epidemiological information that is needed for the process.

\section{ACKNOWLEDGMENTS}

We wish to thank Michael J. Brennan, M.D., for introducing us to this subject originally and for encouragement. Research was supported from 1967 to 1971 by Contract PH-43-67-737 from the Division of Regional Medical Programs of the National Institutes of Health, and preliminary results were reported in Ref. [2]. From 1975 to 1977 support by Contract NO-1-CB-53907 from the National Cancer Institute to the Michigan Cancer Foundation, together with a sabbatical stay at the Cancer Control Agency of British Columbia, made it possible to analyze records from the latter program. We wish to thank those investigators for making that data available, especially David A. Boyes, M.D., and the latter for helpful advice. Computer time for this paper was provided by the University of Michigan. Individuals or organizations interested in using, copying or adapting the computer program should contact the author.

\section{REFERENCES}

1. Gray, L. A. The frequency of taking cervical smears, Obstet. Gynecol. Surv. 24 (7), 909 (1969).

2. Galliher, H. P. Quantitative methodology for medical management, in The recognition of systems in health services, Operations Research Society of America, Baltimore (1969).

3. Fidler, H. K., Boyes, D. A., and Worth, A. J. Cervical cancer detection in British Columbia, J. Obstet. Gynecol. Brit. Commonw. 75, 392 (1968).

4. Cooper, B. S., and Rice, D. P. The economic cost of illness revisited, Bull. U.S. Social Security Administr. Feb. (1976). 